Pacific

Journal of

Mathematics

COMPLETE EMBEDDINGS OF THE COHEN ALGEBRA INTO THREE FAMILIES OF C.C.C., NON-MEASURABLE BOOLEAN ALGEBRAS

NATASHa Dobrinen

Volume $214 \quad$ No. 2

April 2004 


\title{
COMPLETE EMBEDDINGS OF THE COHEN ALGEBRA INTO THREE FAMILIES OF C.C.C., NON-MEASURABLE BOOLEAN ALGEBRAS
}

\author{
NATASHA DobRINEN
}

\begin{abstract}
The Cohen algebra embeds as a complete subalgebra into three classic families of complete, atomless, c.c.c., non-measurable Boolean algebras; namely, the families of Argyros algebras and Galvin-Hajnal algebras, and the atomless part of each Gaifman algebra. It immediately follows that the weak $(\omega, \omega)$-distributive law fails everywhere in each of these Boolean algebras.
\end{abstract}

\section{Introduction.}

Von Neumann conjectured that the countable chain condition and the weak $(\omega, \omega)$-distributive law characterize measurable algebras among Boolean $\sigma$ algebras [Mau]. Consistent counter-examples have been obtained by Maharam [Mah], Jensen [J], Glówczyński [Gl], and Veličković [V]. However, whether von Neumann's proposed characterization of measurable algebras fails within ZFC remains an open problem.

In searching for possible counter-examples to von Neumann's proposed characterization of measurable algebras within ZFC, we investigated three families of complete, c.c.c., non-measurable Boolean algebras, namely, the Argyros, Galvin-Hajnal, and Gaifman algebras, to find out whether these Boolean algebras sustain any weak form of distributivity. By the Cohen algebra we mean the completion of any countable, atomless Boolean algebra. By the $\kappa$-Cohen algebra we mean the completion of the free Boolean algebra on $\kappa$-many generators. We found the following:

\section{Theorem 1.1.}

1) It is possible to construct both atomless Gaifman algebras and Gaifman algebras with atoms.

2) The $c f\left(2^{\omega}\right)$-Cohen algebra embeds as a complete subalgebra into each Galvin-Hajnal algebra.

3) The Cohen algebra embeds as a complete subalgebra into each Argyros algebra and related variants, and into the atomless part of each Gaifman algebra.

We show 1) in $\S 4,2)$ in $\S 2$, and 3$)$ in $\S \S 3$ and 5 . 
Throughout this paper, let $\mathbf{B}$ denote a Boolean algebra and $\mathbf{P}$ denote a partial ordering. Let $\mathbf{B}^{+}$denote $\mathbf{B} \backslash\{\mathbf{0}\}$. Galvin and Hajnal, and Argyros constructed families of partial orderings and Gaifman constructed a family of Boolean algebras to establish strict implications between various chain conditions, including the c.c.c., the $\sigma$-bounded c.c., and $\operatorname{CUP}\left(\mathbf{B}^{+}\right)$. Recall the following definitions:

Definition $1.2([\mathbf{K o}])$. $\mathbf{P}$ satisfies the countable chain condition (c.c.c.) if for each pairwise incompatible subset $X \subseteq \mathbf{P},|X| \leq \omega$.

Definition $1.3([\mathbf{F}])$. $\mathbf{P}$ satisfies the $\sigma$-bounded chain condition ( $\sigma$-bounded c.c.) if there exist subsets $X_{n} \subseteq \mathbf{P}, n<\omega$, such that $\mathbf{P}=\bigcup_{n<\omega} X_{n}$, where $\forall n<\omega$ each pairwise incompatible subset of $X_{n}$ has cardinality $\leq n+1$.

Definition 1.4 ([GP]). Let $S \subseteq \mathbf{P}$ be a nonempty set. For $s_{0}, \ldots, s_{n} \in S$, not necessarily distinct, let

$$
\alpha^{*}\left(s_{0}, \ldots, s_{n}\right)=\frac{1}{n+1} \max \left\{|I|: I \subseteq n+1, \exists p \in \mathbf{P} \forall i \in I s_{i} \geq p\right\} .
$$

The intersection number of $S$ is

$$
\alpha(S)=\inf \left\{\alpha^{*}\left(s_{0}, \ldots, s_{n}\right): n \in \omega, s_{0}, \ldots, s_{n} \in S\right\} .
$$

We say that $\operatorname{CUP}(\mathbf{P})$ holds if $\mathbf{P}$ is a countable union of subsets of $\mathbf{P}$, each of which has positive intersection number; i.e., there exist subsets $X_{n} \subseteq \mathbf{P}$, $n<\omega$, such that $\mathbf{P}=\bigcup_{n<\omega} X_{n}$ and $\forall n<\omega, \alpha\left(X_{n}\right)>0$.

The above three notions are defined for a Boolean algebra $\mathbf{B}$ by replacing $\mathbf{P}$ with $\mathbf{B}^{+}$in Definitions 1.2-1.4. Note that for a partial ordering $\mathbf{P}$, the above three notions are preserved under completions. That is, $\mathbf{P}$ satisfies the c.c.c. ( $\sigma$-bounded c.c.) iff r.o. $(\mathbf{P})$ satisfies the c.c.c. $(\sigma$-bounded c.c. $)$, respectively, and $\operatorname{CUP}(\mathbf{P})$ holds iff $\operatorname{CUP}\left(\right.$ r.o. $\left.(\mathbf{P})^{+}\right)$holds.

It is easy to see that $\operatorname{CUP}(\mathbf{P})$ implies the $\sigma$-bounded c.c., which in turn implies the c.c.c. Gaifman showed that $\operatorname{CUP}(\mathbf{P})$ is strictly stronger than the $\sigma$-bounded c.c. $[\mathbf{G a}]$. Galvin and Hajnal showed that the $\sigma$-bounded c.c. is strictly stronger than the c.c.c. [CN].

Definition 1.5 ([Ko]). B satisfies the weak $(\omega, \omega)$-distributive law (weak $(\omega, \omega)$-d.1. $)$ if for each family $\left(b_{i j}\right)_{i<\omega, j<\omega}$ of elements of $\mathbf{B}$,

$$
\bigwedge_{i<\omega} \bigvee_{j<\omega} b_{i j}=\bigvee_{f: \omega \rightarrow \omega} \bigwedge_{i<\omega} \bigvee_{j \leq f(i)} b_{i j}
$$

provided that $\bigvee_{j<\omega} b_{i j}$ for each $i<\omega, \bigwedge_{i<\omega} \bigvee_{j<\omega} b_{i j}$, and $\bigwedge_{i<\omega} \bigvee_{j \leq f(i)} b_{i j}$ for each $f: \omega \rightarrow \omega$ exist in $\mathbf{B}$. We say that the weak $(\omega, \omega)$-d.l. fails everywhere in $\mathbf{B}$ if there exist $\left(b_{i j}\right)_{i<\omega, j<\omega} \subseteq \mathbf{B}$ such that $\bigwedge_{i<\omega} \bigvee_{j<\omega} b_{i j}=\mathbf{1}$ and $\bigvee_{f: \omega \rightarrow \omega} \bigwedge_{i<\omega} \bigvee_{j \leq f(i)} b_{i j}=\mathbf{0}$ 
Definition 1.6 ([Ko]). A Boolean $\sigma$-algebra $\mathbf{B}$ is measurable if there exists a function $\mu: \mathbf{B} \rightarrow[0, \infty)$ which is strictly positive $(\mu(b)=0 \leftrightarrow b=\mathbf{0})$ and $\sigma$-additive $\left(\mu\left(\bigvee_{i<\omega} b_{i}\right)=\Sigma_{i<\omega} \mu\left(b_{i}\right)\right.$ for all pairwise disjoint $\left\{b_{i}: i<\omega\right\} \subseteq$ B).

Theorem 1.7 ([Ko]). Every measurable Boolean algebra $\mathbf{B}$ satisfies the weak $(\omega, \omega)$-d.l. and the c.c.c.

Theorem 1.8 (Kelley, $[\mathbf{K e}])$. $\operatorname{CUP}\left(\mathbf{B}^{+}\right)$holds iff $\mathbf{B}$ carries a strictly positive, finitely-additive measure $\mu: \mathbf{B} \rightarrow[0, \infty)$.

The following Theorem 1.9 of Kelley completely characterizes measurable algebras among Boolean $\sigma$-algebras in ZFC by strengthening the c.c.c. to $\operatorname{CUP}\left(\mathbf{B}^{+}\right)$.

Theorem 1.9 (Kelley, $[\mathbf{K e}]$ ). If $\mathbf{B}$ is a $\sigma$-algebra, then $\mathbf{B}$ is measurable iff $\mathbf{B}$ satisfies the weak $(\omega, \omega)$-d.l. and $\operatorname{CUP}\left(\mathbf{B}^{+}\right)$holds.

Theorem 1.10 (Folklore). The weak $(\omega, \omega)$-d.l. fails everywhere in the Cohen algebra.

Each of our complete embeddings of the Cohen algebra will involve the following notions and lemmas:

Definition 1.11 ([Ko]). A subalgebra $\mathbf{A}$ of a Boolean algebra $\mathbf{B}$ is a regular subalgebra of $\mathbf{B}$ if for each $M \subseteq \mathbf{A}$ such that $\bigvee^{\mathbf{A}} M$ exists in $\mathbf{A}, \bigvee^{\mathbf{B}} M$ exists in $\mathbf{B}$ and $\bigvee^{\mathbf{A}} M=\bigvee^{\mathbf{B}} M$.

Lemma 1.12. Let $\mathbf{B}$ be a Boolean algebra, $\mathbf{P}$ a dense subset of $\mathbf{B}^{+}$, and $\mathbf{A}$ a subalgebra of $\mathbf{B}$. A is a regular subalgebra of $\mathbf{B}$ iff $\forall p \in \mathbf{P} \exists a_{p} \in \mathbf{A}^{+}$such that whenever $a \in \mathbf{A}$ and $a_{p} \wedge a \neq \mathbf{0}$, then $p \wedge a \neq \mathbf{0}$.

Definition 1.13 ([Ko]). A subalgebra $\mathbf{A}$ of a Boolean algebra $\mathbf{B}$ is a complete subalgebra of $\mathbf{B}$ if for each subset $M \subseteq \mathbf{A}$ such that $\bigvee^{\mathbf{B}} M$ exists, $\bigvee^{\mathbf{A}} M$ exists and $\bigvee^{\mathbf{B}} M=\bigvee^{\mathbf{A}} M$.

Definition 1.14 ([Ko]). A monomorphism $f: \mathbf{A} \rightarrow \mathbf{B}$ is complete if for each $M \subseteq \mathbf{A}$ for which $\bigvee^{\mathbf{A}} M$ exists, $f\left(\bigvee^{\mathbf{A}} M\right)=\bigvee^{\mathbf{B}}\{f(b): b \in M\}$.

The following lemma is a natural consequence of the Sikorski Extension Theorem:

Lemma $1.15([\mathbf{K o}])$. If $\mathbf{B}$ is a complete Boolean algebra and $\mathbf{A}$ is a regular subalgebra of $\mathbf{B}$, then there is a complete monomorphism from r.o. $\left(\mathbf{A}^{+}\right)$into B.

Throughout this paper, let $\mathbf{P}_{G H}, \mathbf{P}_{A}$, and $\mathbf{B}_{G}$ denote members of the families of Galvin-Hajnal partial orderings, Argyros partial orderings, and Gaifman algebras, respectively. Galvin and Hajnal, Argyros, and Gaifman 
showed that in r.o. $\left(\mathbf{P}_{G H}\right)$, r.o. $\left(\mathbf{P}_{A}\right)$, and $\mathbf{B}_{G}$, respectively, $\operatorname{CUP}\left(\mathbf{B}^{+}\right)$fails; thus, by Kelley's Theorem 1.9, these three Boolean algebras are not measurable. However, this is not the only reason measurability fails in these algebras. By Theorem 1.10, completely embedding the Cohen algebra into r.o. $\left(\mathbf{P}_{G H}\right)$, r.o. $\left(\mathbf{P}_{A}\right)$, and the atomless part of r.o. $\left(\mathbf{B}_{G}{ }^{+}\right)$shows that no weak form of distributivity holds in these Boolean algebras.

Remark 1.16. We thank the referee for pointing out the following: By a result of Shelah, in c.c.c. Suslin forcings, adding a Cohen real is equivalent to the weak $(\omega, \omega)$-d.l. failing everywhere $[\mathbf{S}]$. Hence, to prove 3$)$ of Theorem 1.1, it would suffice to show that the weak $(\omega, \omega)$-d.l. fails everywhere in the Argyros and Gaifman algebras. However, Shelah's result does not apply to the Galvin-Hajnal algebra, since $\mathbf{P}_{G H}$ is not Suslin.

For $\kappa=\operatorname{cf}\left(2^{\omega}\right)$ in the case of Galvin-Hajnal algebras, and for $\kappa=\omega$ in the case of Argyros algebras and the atomless part of Gaifman algebras, the method we employ for completely embedding the $\kappa$-Cohen algebra is the following: Choose $\kappa$ many independent elements $\left\{c_{i}: i<\kappa\right\} \subseteq \mathbf{B}$ in such a way that the subalgebra $\mathbf{C}_{\kappa}$ generated by $\left\{c_{i}: i<\kappa\right\}$ satisfies the conditions of Lemma 1.12. Then $\mathbf{C}_{\kappa}$ is isomorphic to the free Boolean algebra on $\kappa$-many generators and is a regular subalgebra of $\mathbf{B}$. Since $\mathbf{B}$ is complete, Lemma 1.15 implies that the completion of $\mathbf{C}_{\kappa}$, the $\kappa$-Cohen algebra, embeds into $\mathbf{B}$ as a complete subalgebra.

\section{A complete embedding of the Cohen algebra into the Galvin-Hajnal algebra.}

Galvin and Hajnal constructed a separative, atomless partial ordering $\mathbf{P}_{G H}$ which satisfies the c.c.c. but not the $\sigma$-bounded c.c. $[\mathbf{C N}]$. To do this, they used the following family of sets:

Recall that for a set $S,[S]^{2}=\{\{\beta, \gamma\}: \beta, \gamma \in S$ and $\beta \neq \gamma\}$, the collection of all two-element subsets of $S$. In this section, we fix a well-ordering on $2^{\omega}$.

Lemma 2.1 (Galvin-Hajnal, $[\mathbf{C N}]$ ). There is a family of sets $\left\{S_{\alpha}: \alpha<\right.$ $\left.2^{\omega}\right\}$ with the following four properties:

$$
\begin{aligned}
& \forall \alpha<2^{\omega} \quad S_{\alpha} \subseteq \alpha \\
& \forall \alpha<2^{\omega} \quad\left[S_{\alpha}\right]^{2} \subseteq \bigcup_{\gamma<2^{\omega}}\left\{\{\beta, \gamma\}: \beta \in S_{\gamma}\right\} \\
& \forall \alpha<2^{\omega} \quad \text { o.t. }\left(S_{\alpha}\right) \leq \omega ; \\
& \text { If } S \subseteq 2^{\omega},[S]^{2} \subseteq \bigcup_{\gamma<2^{\omega}}\left\{\{\beta, \gamma\}: \beta \in S_{\gamma}\right\}, \text { and o.t. }(S) \leq \omega \\
& \text { then } \exists \alpha<2^{\omega} \text { such that } S=S_{\alpha} .
\end{aligned}
$$

The following properties of the collection of sets $\left\{S_{\alpha}: \alpha<2^{\omega}\right\}$ will be used extensively: 
Lemma 2.2. Suppose $\eta, \zeta$ are ordinals with $\eta, \zeta<2^{\omega}$ and $\left|S_{\eta}\right|=\omega$. Then there exist $\zeta<\alpha<\beta<2^{\omega}$ such that $S_{\alpha} \cup S_{\beta}=S_{\eta}$.

Proof. Let $\mathcal{X}=\left\{S_{\alpha}: \alpha \leq \zeta, S_{\alpha} \subseteq S_{\eta}\right\} \cup\left\{S_{\eta} \backslash S_{\alpha}: \alpha \leq \zeta\right\}$. $|\mathcal{X}|<2^{\omega}$, so choose some $S \subseteq S_{\eta}$ such that $S \notin \mathcal{X}$ and $S_{\eta} \backslash S \notin \mathcal{X}$. $[S]^{2},\left[S_{\eta} \backslash S\right]^{2} \subseteq$ $\left[S_{\eta}\right]^{2} \subseteq \bigcup_{\alpha<2^{\omega}}\left\{\{\beta, \alpha\}: \beta \in S_{\alpha}\right\}$, by (S2). (S4) implies $\exists \alpha, \beta<2^{\omega}$ such that $S_{\alpha}=S$ and $S_{\beta}=S_{\eta} \backslash S . \alpha, \beta>\zeta$, since $S \notin \mathcal{X}$.

Lemma 2.3. Given $\alpha_{0}<2^{\omega}$, there is a sequence $\alpha_{0}<\alpha_{1}<\alpha_{2}<\cdots<$ $\lambda<2^{\omega}$ of order type $\omega+1$ such that for each $i<\omega$,

$$
\begin{aligned}
S_{\alpha_{i+1}} & =\left\{\alpha_{0}, \ldots, \alpha_{i}\right\} ; \\
S_{\lambda} & =\left\{\alpha_{i}: 0<i<\omega\right\} .
\end{aligned}
$$

Proof. Let $\alpha_{0}<2^{\omega}$. $\left[\left\{\alpha_{0}\right\}\right]^{2}=\emptyset$, so $\exists \alpha_{1}<2^{\omega}$ for which $S_{\alpha_{1}}=\left\{\alpha_{0}\right\}$, by (S4). (S1) implies $\alpha_{1}>\alpha_{0}$. Given $\alpha_{0}<\cdots<\alpha_{n}$ where for each $0<j \leq n$ $S_{\alpha_{j}}=\left\{\alpha_{0}, \ldots, \alpha_{j-1}\right\}$, the set $\left[\left\{\alpha_{0}, \ldots, \alpha_{n}\right\}\right]^{2} \subseteq \bigcup_{\alpha<2^{\omega}}\left\{\{\beta, \alpha\}: \beta \in S_{\alpha}\right\}$. By (S1) and (S4), there is some $\alpha_{n+1}>\alpha_{n}$ such that $S_{\alpha_{n+1}}=\left\{\alpha_{0}, \ldots, \alpha_{n}\right\}$.

By our choice of the $\alpha_{n},\left[\left\{\alpha_{1}, \alpha_{2}, \ldots\right\}\right]^{2} \subseteq \bigcup_{\alpha<2}\left\{\{\beta, \alpha\}: \beta \in S_{\alpha}\right\}$. By (S1) and (S4), there is a $\lambda<2^{\omega}$ such that $S_{\lambda}=\left\{\alpha_{i}: 0<i<\omega\right\}$ and $\lambda>\alpha_{j}$ for all $j<\omega$.

Galvin and Hajnal constructed the following partial ordering $\mathbf{P}_{G H}: \forall \alpha<$ $2^{\omega}$, let

$$
V_{\alpha}=\left\{f: 2^{\omega} \rightarrow 2: \forall \beta \in S_{\alpha}(f(\beta)=0), \text { and } f(\alpha)=1\right\} .
$$

That is, $V_{\alpha}$ is the collection of all functions from $2^{\omega}$ into 2 which send each element of $S_{\alpha}$ to 0 and send $\alpha$ to 1 . Let

$$
\mathbf{P}_{G H}=\left\{\bigcap_{\alpha \in F} V_{\alpha}: F \in\left[2^{\omega}\right]^{<\omega} \text { and } \bigcap_{\alpha \in F} V_{\alpha} \neq \emptyset\right\} .
$$

$\mathbf{P}_{G H}$ is the collection of nonempty intersections of finitely many of the $V_{\alpha}$ 's, partially ordered by inclusion.

Theorem 2.4 (Galvin-Hajnal, $[\mathbf{C N}]) \cdot\left\langle\mathbf{P}_{G H}, \subseteq\right\rangle$ is a separative, atomless partial ordering which satisfies the c.c.c., but not the $\sigma$-bounded c.c.

Let $e: \mathbf{P}_{G H} \rightarrow$ r.o. $\left(\mathbf{P}_{G H}\right)$ denote the canonical embedding of $\mathbf{P}_{G H}$ into its completion r.o. $\left(P_{G H}\right)$. We shall call r.o. $\left(\mathbf{P}_{G H}\right)$ the Galvin-Hajnal algebra. Since the $\sigma$-bounded c.c. fails in $P_{G H}, \operatorname{CUP}\left(\right.$ r.o. $\left.\left(\mathbf{P}_{G H}\right)^{+}\right)$fails. By Kelley's Theorem 1.9, r.o. $\left(\mathbf{P}_{G H}\right)$ is not measurable.

Theorem 2.5. The $c f\left(2^{\omega}\right)$-Cohen algebra embeds as a complete subalgebra into r.o. $\left(\mathbf{P}_{G H}\right)$. 
Proof. Let $\kappa$ denote $\operatorname{cf}\left(2^{\omega}\right)$. We construct a subalgebra $\mathbf{C}_{\kappa}$ of r.o. $\left(\mathbf{P}_{G H}\right)$ and show that $\mathbf{C}_{\kappa}$ is isomorphic to the free Boolean algebra on $\kappa$-many generators, and is a regular subalgebra of r.o. $\left(\mathbf{P}_{G H}\right)$.

Construction of $\mathbf{C}_{\kappa}$ : By Lemma 2.3, there exist $\kappa$-many sequences, each of order type $\omega+1$, of the form $\alpha(i, 0)<\alpha(i, 1)<\alpha(i, 2)<\cdots<\alpha(i, j)<$ $\alpha(i, j+1)<\cdots<\lambda(i), i<\kappa$, such that the following hold: $\forall i<\kappa, \forall j<$ $j^{\prime}<\omega, \alpha(i, j)<\alpha\left(i, j^{\prime}\right)<\lambda(i) ; \forall i<i^{\prime}<\kappa, \lambda(i)<\alpha\left(i^{\prime}, 0\right) ;$ and $\forall i<$ $\kappa, \forall 0<j<\omega$,

$$
\begin{aligned}
S_{\alpha(i, j)} & =\{\alpha(i, k): k<j\} \\
S_{\lambda(i)} & =\{\alpha(i, j): 0<j<\omega\} .
\end{aligned}
$$

Note that the sets $\{\alpha(i, j): j<\omega\} \cup\{\lambda(i)\}, i<\kappa$, are pairwise disjoint. For each $i<\kappa$, let

$$
c_{i}=\bigvee_{0<j<\omega} e\left(V_{\alpha(i, j)}\right)
$$

in r.o. $\left(\mathbf{P}_{G H}\right)$. Let

$$
\mathbf{C}_{\kappa}=\left\langle c_{i}: i<\kappa\right\rangle,
$$

the subalgebra of r.o. $\left(\mathbf{P}_{G H}\right)$ generated by $\left\{c_{i}: i<\kappa\right\}$. The elements $V_{\lambda(i)}, i<\kappa$, will be used in Proposition 2.11 to ensure that the generators of $\mathbf{C}_{\kappa}$ are independent. The following simple facts will be useful:

Fact 2.6. $\forall p, q \in \mathbf{P}_{G H}, e(p) \wedge e(q)=e(p \cap q)$.

Fact 2.7. For each finite $F \subseteq 2^{\omega}, \bigcap_{\alpha \in F} V_{\alpha} \neq \emptyset$ iff $\left(\bigcup_{\alpha \in F} S_{\alpha}\right) \cap F=\emptyset$.

Fact 2.8. If $\left\{p_{i}: i<\omega\right\} \subseteq \mathbf{P}_{G H}$ is infinite, then $\bigcap_{i<\omega} e\left(p_{i}\right)=\emptyset$.

If $\left\{p_{i}: i<\omega\right\}$ is infinite and $q \in \bigcap_{i<\omega} e\left(p_{i}\right)$, then every $f \in q$ must take infinitely many elements of $2^{\omega}$ to 1 . There are no such $q \in \mathbf{P}_{G H}$.

Fact 2.9. Given $i<\kappa$, if $F$ is a finite subset of $2^{\omega}$ and $\bigcup_{\alpha \in F} S_{\alpha} \supseteq S_{\lambda(i)}$, then $e\left(\bigcap_{\alpha \in F} V_{\alpha}\right) \leq-c_{i}$. In particular, $\forall i<\kappa, e\left(V_{\lambda(i)}\right) \leq-c_{i}$.

Suppose $\bigcup_{\alpha \in F} S_{\alpha} \supseteq S_{\lambda(i)}$. Then $\forall p \in e\left(\bigcap_{\alpha \in F} V_{\alpha}\right), \forall f \in p, \forall 0<j<\omega$, $f(\alpha(i, j))=0$. However, $\forall q \in c_{i}, \exists f \in q$ and $\exists 0<j<\omega$ such that $f(\alpha(i, j))=1$. So $e\left(\bigcap_{\alpha \in F} V_{\alpha}\right) \cap c_{i}=\emptyset$.

Fact 2.10. $\forall i<\kappa, c_{i}=\bigcup_{0<j<\omega} e\left(V_{\alpha(i, j)}\right)$.

Proof. Clearly, $c_{i} \supseteq \bigcup_{0<j<\omega} e\left(V_{\alpha(i, j)}\right)$. Suppose $p \notin \bigcup_{0<j<\omega} e\left(V_{\alpha(i, j)}\right)$. Let $F$ be the finite subset of $2^{\omega}$ such that $p=\bigcap_{\alpha \in F} V_{\alpha} . \forall 0<j<\omega, p \nsubseteq V_{\alpha(i, j)}$, so $S_{\lambda(i)} \cap F=\emptyset$. By Lemma $2.2, \exists \gamma<\beta<2^{\omega}$ with $\gamma>\sup (F \cup\{\lambda(i)\})$ such that $S_{\gamma} \cup S_{\beta}=S_{\lambda(i)}$. By Fact 2.7, $V_{\gamma} \cap V_{\beta} \cap p \neq \emptyset$. By Fact 2.9, $e\left(V_{\gamma} \cap V_{\beta} \cap p\right) \subseteq-c_{i}$, so $e(p) \nsubseteq c_{i}$. Since $c_{i}$ is an open subset of $\mathbf{P}_{G H}$, $e(p) \not c_{i}$ implies $p \notin c_{i}$. Thus, $c_{i} \subseteq \bigcup_{0<j<\omega} e\left(V_{\alpha(i, j)}\right)$. 
Next we show that the generators of $\mathbf{C}_{\kappa}$ are independent.

Proposition 2.11. For finite sets $I, J \subseteq \kappa, \bigwedge_{i \in I} c_{i} \wedge \bigwedge_{j \in J}-c_{j}=\mathbf{0}$ iff $I \cap J \neq \emptyset$.

Proof. Let $I, J \subseteq \kappa$ be disjoint, finite sets, and let $r=\bigcap_{i \in I} V_{\alpha(i, 1)} \cap$ $\bigcap_{j \in J} V_{\lambda(j)}$. Fact 2.7 implies $r \neq \emptyset$. By Fact 2.9, $\bigwedge_{i \in I} c_{i} \wedge \bigwedge_{j \in J}-c_{j} \geq$ $\bigwedge_{i \in I} e\left(V_{\alpha(i, 1)}\right) \wedge \bigwedge_{j \in J} e\left(V_{\lambda(j)}\right)=e(r)>\mathbf{0}$.

Since $\mathbf{C}_{\kappa}$ is generated by $\operatorname{cf}\left(2^{\omega}\right)$-many independent elements, $\mathbf{C}_{\kappa}$ is isomorphic to the free Boolean algebra on $\operatorname{cf}\left(2^{\omega}\right)$-many generators.

The next proposition will aid us in constructing elements $c_{p}$ satisfying the conditions of Lemma 1.12.

Proposition 2.12. Given $p \in \mathbf{P}_{G H}$, there are at most finitely many $i<\kappa$ for which either $e(p) \wedge c_{i}=\mathbf{0}$ or $e(p) \wedge-c_{i}=\mathbf{0}$.

Proof. Suppose $p \in \mathbf{P}_{G H}$ and let $J \subseteq \kappa$ be defined by $j \in J \leftrightarrow e(p) \wedge c_{j}=\mathbf{0}$. $\forall j \in J$,

$$
\mathbf{0}=e(p) \wedge c_{j}=e(p) \cap\left(\bigcup_{0<k<\omega} e\left(V_{\alpha(j, k)}\right)\right)=\bigcup_{0<k<\omega}\left(e(p) \cap e\left(V_{\alpha(j, k)}\right)\right),
$$

the second equality following from Fact 2.10. Thus, $\forall j \in J, \forall 0<k<$ $\omega, p \cap V_{\alpha(j, k)}=\emptyset$.

Let $F$ be the finite subset of $2^{\omega}$ such that $p=\bigcap_{\alpha \in F} V_{\alpha}$. The following can be shown by an easy induction argument using Fact 2.7: If $e(p) \wedge c_{j}=\mathbf{0}$, then either $\alpha(j, 0) \in F$ or $S_{\lambda(j)} \subseteq \bigcup_{\alpha \in F} S_{\alpha}$.

$\alpha(j, 0) \in F$ for at most finitely many $j<\kappa$, since $F$ is finite. By (S3), o.t. $\left(\bigcup_{\alpha \in F} S_{\alpha}\right)<\omega \cdot \omega$, so $S_{\lambda(j)} \subseteq \bigcup_{\alpha \in F} S_{\alpha}$ for at most finitely many $j<\kappa$. Hence, $e(p) \wedge c_{j}=\mathbf{0}$ for at most finitely many $j<\kappa$. Thus, $|J|<\omega$.

Let $I \subseteq \kappa$ be defined by $i \in I \leftrightarrow e(p) \wedge-c_{i}=\mathbf{0}$. Then

$$
e(p) \subseteq \bigcap_{i \in I} c_{i}=\bigcap_{i \in I} \bigcup_{0<k<\omega} e\left(V_{\alpha(i, k)}\right)=\bigcup_{g: I \rightarrow \omega \backslash\{0\}} \bigcap_{i \in I} e\left(V_{\alpha(i, g(i))}\right),
$$

where the first equality follows from Fact 2.10. If $I$ is infinite, then Fact 2.8 implies that $\forall g: I \rightarrow \omega \backslash\{0\}, \bigcap_{i \in I} e\left(V_{\alpha(i, g(i))}\right)=\emptyset$, contradicting $p \neq \emptyset$. Thus, $|I|<\omega$.

Now we use Proposition 2.12 to show that $\mathbf{C}_{\kappa}$ satisfies the conditions of Lemma 1.12.

Proposition 2.13. $\forall p \in \mathbf{P}_{G H} \exists c_{p} \in \mathbf{C}_{\kappa}{ }^{+}$such that $\forall c \in \mathbf{C}_{\kappa}$, if $c_{p} \wedge c \neq \mathbf{0}$, then $e(p) \wedge c \neq \mathbf{0}$. 
Proof. Fix $p \in \mathbf{P}_{G H}$ and let $F$ be the finite subset of $2^{\omega}$ such that $p=$ $\bigcap_{\alpha \in F} V_{\alpha}$. Let $I, J \subseteq \kappa$ be the finite disjoint sets of Proposition 2.12. Let $c_{p}=\left(\bigwedge_{i \in I} c_{i}\right) \wedge\left(\bigwedge_{j \in J}-c_{j}\right) . c_{p} \in \mathbf{C}_{\kappa}{ }^{+}$, by Proposition 2.11. ( $c_{p}$ is actually the minimal cover for $e(p)$ in $\mathbf{C}$.)

Suppose $c=\left(\bigwedge_{k \in K} c_{k}\right) \wedge\left(\bigwedge_{l \in L}-c_{l}\right) \in \mathbf{C}_{\kappa}$ and $c_{p} \wedge c \neq \mathbf{0}$. Then $I \cap$ $L=J \cap K=\emptyset . \quad J \cap K=\emptyset$ implies $\forall k \in K, \exists 0<m_{k}<\omega$ such that $p \cap V_{\alpha\left(k, m_{k}\right)} \neq \emptyset$. By Fact 2.7,

$$
\left(F \cup\left\{\alpha\left(k, m_{k}\right): k \in K\right\}\right) \bigcap\left(\bigcup_{\alpha \in F} S_{\alpha} \cup\left\{\alpha(k, m): k \in K, m<m_{k}\right\}\right)=\emptyset .
$$

$I \cap L=\emptyset$ implies $\forall l \in L, e(p) \not \subset c_{l}$. Thus, $\forall l \in L, \forall 0<n<\omega, e(p) \not \leq$ $e\left(V_{\alpha(l, n)}\right)$, which implies $\alpha(l, n) \notin F$. Therefore,

$$
F \bigcap\left(\bigcup_{l \in L} S_{\lambda(l)}\right)=\emptyset \text {. }
$$

Using Lemma 2.2, $\forall l \in L$ choose $\beta_{l}, \alpha_{l}>\sup (F \cup\{\lambda(n): n \in K \cup L\})$ such that $S_{\alpha_{l}} \cup S_{\beta_{l}}=S_{\lambda(l)}$. Let

$$
r=\bigcap_{k \in K} V_{\alpha\left(k, m_{k}\right)} \cap \bigcap_{l \in L}\left(V_{\alpha_{l}} \cap V_{\beta_{l}}\right) .
$$

$r \neq \emptyset$, by Fact 2.7. $e(r) \leq c$, by Fact 2.9. Furthermore, $r \cap p \neq \emptyset$, by Fact $2.7,(2.9),(2.10)$, and the fact that $\forall l \in L, \alpha_{l}, \beta_{l}$ are larger than $\sup (F \cup\{\lambda(n): n \in K \cup L\})$. Hence, $\mathbf{0}<e(r \cap p) \leq c$, so $e(p) \wedge c \neq \mathbf{0}$.

It follows from Lemma 1.12 and Proposition 2.13 that $\mathbf{C}_{\kappa}$ is a regular subalgebra of r.o. $\left(\mathbf{P}_{G H}\right)$. Thus, by Lemma 1.15 , r.o. $\left(\mathbf{C}_{\kappa}{ }^{+}\right)$, the $\kappa$-Cohen algebra, embeds into r.o. $\left(\mathbf{P}_{G H}\right)$ as a complete subalgebra. That is, $\mathbf{P}_{G H}$ adds $\operatorname{cf}\left(2^{\omega}\right)$-many side-by-side Cohen reals.

\section{A complete embedding of the Cohen algebra into the Argyros algebra.}

Agyros constructed a separative, atomless partial ordering $\mathbf{P}_{A}$ in which the $\sigma$-bounded c.c. holds but $\operatorname{CUP}\left(\mathbf{P}_{A}\right)$ fails, and, assuming $\mathrm{CH}$, property $K_{3}$ also fails $[\mathbf{A}]$. He constructed $\mathbf{P}_{A}$ using three basic types of elements. In this section, let $2^{\omega}$ denote the set of functions from $\omega$ to 2 . For $X, Y \in[\omega]^{<\omega}$, let

$$
\begin{aligned}
& B_{X}=\left\{f \in 2^{\omega}: \forall x \in X f(x)=1\right\}, \\
& \bar{B}_{Y}=\left\{f \in 2^{\omega}: \forall y \in Y \quad f(y)=0\right\} .
\end{aligned}
$$

For the third type of element, Argyros constructed a tree $T \subseteq[\omega]^{2}$ as follows: Let $\left\{S_{n m}: n<\omega, 1 \leq m \leq 3^{n}\right\}$ be a family of sets such that $\forall n, m<\omega$, 
$S_{n m} \in[\omega]^{3}$ and $S_{n m} \cap S_{n^{\prime} m^{\prime}}=\emptyset$ whenever $\langle n, m\rangle \neq\left\langle n^{\prime}, m^{\prime}\right\rangle$. For each $n<\omega$, let $\operatorname{Lev}(n)=\bigcup_{1 \leq m \leq 3^{n}}\left[S_{n m}\right]^{2}$. For each $n<\omega$, index the elements of $\operatorname{Lev}(n)$ as $s_{n j}, 1 \leq j \leq 3^{n+1}$. The partial ordering on $T$ is defined at level $n+1$ as follows: For $s=s_{n j} \in \operatorname{Lev}(n)$ and $t \in \operatorname{Lev}(n+1), s \prec t \leftrightarrow t \in\left[S_{n+1, j}\right]^{2}$. Let $T=\bigcup_{n<\omega} \operatorname{Lev}(n) .\langle T, \prec\rangle$ is the Argyros tree.

For $s=\{k, l\} \in T$, let

$$
K_{s}=\left(B_{\{k\}} \cap \bar{B}_{\{l\}}\right) \cup\left(B_{\{l\}} \cap \bar{B}_{\{k\}}\right) .
$$

$K_{s}$ is the set of all functions in $2^{\omega}$ which are nonconstant on $s$. Let $\mathcal{B r}$ be the set of all branches (finite and infinite) of $T$. For $\sigma \in \mathcal{B} r$, let

$$
A_{\sigma}=\bigcap_{s \in \sigma} K_{s} .
$$

$A_{\sigma}$ is the set of all functions in $2^{\omega}$ which are nonconstant on every node $s \in \sigma$. Let

$$
\mathbf{P}_{A}=\left\{B_{X} \cap \bar{B}_{Y} \cap \bigcap_{\sigma \in \Sigma} A_{\sigma}: X, Y \in[\omega]^{<\omega} \text { and } \Sigma \in[\mathcal{B} r]^{<\omega}\right\} \backslash\{\emptyset\},
$$

the collection of all nonempty intersections of finitely many elements of the three forms $B_{X}, \bar{B}_{Y}$, and $A_{\sigma}$, partially ordered by inclusion. We call $\left\langle\mathbf{P}_{A}, \subseteq\right\rangle$ the Argyros partial ordering.

Theorem 3.1 (Argyros, $[\mathbf{A}])$. $\left\langle\mathbf{P}_{A}, \subseteq\right\rangle$ is a separative, atomless partial ordering which satisfies the $\sigma$-bounded c.c. but not $\mathrm{CUP}\left(\mathbf{P}_{A}\right)$, and, assuming $\mathrm{CH}$, does not satisfy property $K_{3}$.

Let $e: \mathbf{P}_{A} \rightarrow$ r.o. $\left(\mathbf{P}_{A}\right)$ be the canonical embedding of $\mathbf{P}_{A}$ into its completion. We shall call r.o. $\left(\mathbf{P}_{A}\right)$ the Argyros algebra. By Argyros' Theorem 3.1, r.o. $\left(\mathbf{P}_{A}\right)$ is a complete, atomless Boolean algebra which satisfies the $\sigma$-bounded c.c. but not $\operatorname{CUP}\left(\right.$ r.o. $\left.\left(\mathbf{P}_{A}\right)^{+}\right)$, and hence, by Kelley's Theorem 1.9 , is not measurable.

Definition 3.2. We will say that $s, t \in T$ are siblings if $s \neq t$ and $\exists v \in T$ such that $s$ and $t$ are both immediate successors of $v . s, t, u \in T$ are triplets if they are pairwise siblings.

Note: If $s$ and $t$ are siblings, then there exist unique $m, n<\omega$ such that $s, t \in\left[S_{m n}\right]^{2}$, and $K_{s} \cap K_{t} \neq \emptyset$. If $s, t, u$ are triplets, then $s=t \triangle u$ (the set-theoretic difference of $t$ and $u$ in $\omega$ ), and $K_{s} \cap K_{t} \cap K_{u}=\emptyset$.

Remark 3.3. The elements of $\mathbf{P}_{A}$ are not uniquely represented by the form $B_{X} \cap \bar{B}_{Y} \cap \bigcap_{\sigma \in \Sigma} A_{\sigma}$. For instance, if $s=\{k, l\} \in \sigma \in \mathcal{B} r$, then $B_{\{k\}} \cap A_{\sigma}=$ $B_{\{k\}} \cap \bar{B}_{\{l\}} \cap A_{\sigma}=\bar{B}_{\{l\}} \cap A_{\sigma}$. We shall hold to the following convention: Given $S \subseteq T$ and $X, Y \in[\omega]<\omega$, the representation $B_{X} \cap \bar{B}_{Y} \cap \bigcap_{s \in S} K_{s}$ of a subset of $2^{\omega}$ is said to be in the normal form if and only if $X \cap Y=\emptyset$, 
$\forall s \in S(s \cap(X \cup Y)=\emptyset)$, and $S$ contains no triplets. If $B_{X} \cap \bar{B}_{Y} \cap \bigcap_{s \in S} K_{s}$ is the normal form representation of some $B_{U} \cap \bar{B}_{V} \cap \bigcap_{\sigma \in \Sigma} A_{\sigma} \in \mathbf{P}_{A}$, then $X \supseteq U, Y \supseteq V, X \cap Y=\emptyset$, and $S \subseteq \bigcup\{\sigma: \sigma \in \Sigma\}$. It is not hard to see that for each element $p \in \mathbf{P}_{A}$ there is a unique normal form representation of $p$. The normal form is not necessary for the proceeding proofs, but rather serves to simplify notation.

Lemma 3.4. Let $p=B_{X} \cap \bar{B}_{Y} \cap \bigcap_{s \in S} K_{s}$ be a subset of $2^{\omega}$, (not necessarily in the normal form, and not necessarily in $\mathbf{P}_{A}$ ). Then $p \neq \emptyset$ iff the following four conditions hold:

(L1 p) $\quad X \cap Y=\emptyset$;

(L2 $p) \quad \forall s \in S, s \nsubseteq X$ and $s \nsubseteq Y$;

(L3 $p$ ) $S$ does not contain any triplets;

(L4 $p) \quad$ If $s, t \in S$ are siblings, then either $(s \triangle t) \cap X=\emptyset$ or $(s \triangle t) \cap Y=\emptyset$.

In particular, if $p=B_{X} \cap \bar{B}_{Y} \cap \bigcap_{s \in S} K_{s}$ is in the normal form, then $p \neq \emptyset$.

Proof. The forward direction is trivial. Assume (L1 $p)-(\mathrm{L} 4 p)$ hold. We show there is a partial function $f_{p}$ with domain $X \cup Y \cup \cup S$ such that every total extension of $f_{p}$ is in $B_{X} \cap \bar{B}_{Y} \cap \bigcap_{s \in S} K_{s}$.

By (L1 $p$ ), $B_{X} \cap \bar{B}_{Y} \neq \emptyset$. By (L3 $p$ ), we can divide $S$ into two disjoint sets: $\Gamma=\{s \in S: s$ has no sibling in $S\}$ and $\Theta=\{s \in S: s$ has exactly one sibling in $S\} . \forall s \in \Gamma$, by (L2 $p$ ) $s \nsubseteq X$ and $s \nsubseteq Y$. Hence, there is a partial function $f_{s}$ such that $\operatorname{dom}\left(f_{s}\right)=X \cup Y \cup s, f_{s} \uparrow X \equiv 1, f_{s} \uparrow Y \equiv 0$, and $f_{s} \uparrow s$ is nonconstant. Every extension of $f_{s}$ to a total function is in $B_{X} \cap \bar{B}_{Y} \cap K_{s}$.

For each pair of siblings $t, u \in \Theta$, there is a partial function $f_{t, u}$ such that $\operatorname{dom}\left(f_{t, u}\right)=X \cup Y \cup t \cup u, f_{t, u} \uparrow X \equiv 1, f_{t, u} \uparrow Y \equiv 0$, and every extension of $f_{t, u}$ to a total function is in $B_{X} \cap \bar{B}_{Y} \cap K_{t} \cap K_{u}$. To define such an $f_{t, u}$ on $t \cup u$ while preserving $f_{t, u} \uparrow X \equiv 1$ and $f_{t, u} \uparrow Y \equiv 0$, we consider 3 cases. If $(t \triangle u) \cap X \neq \emptyset$, then $(t \cap u) \cap X=\emptyset$ by (L2 $p$ ), and $(t \triangle u) \cap Y=\emptyset$ by (L4 $p$ ); so let $f_{t, u} \uparrow(t \triangle u) \equiv 1$ and $f_{t, u} \uparrow(t \cap u) \equiv 0$. If $(t \cap u) \cap Y \neq \emptyset$, then by (L2 p) $(t \triangle u) \cap Y=\emptyset$; so let $f_{t, u} \uparrow(t \triangle u) \equiv 1$ and $f_{t, u} \uparrow(t \cap u) \equiv 0$. If $(t \triangle u) \cap X$ and $(t \cap u) \cap Y=\emptyset$, let $f_{t, u} \uparrow(t \triangle u) \equiv 0$ and $f_{t, u} \uparrow(t \cap u) \equiv 1$.

Since two elements of $S$ have nonempty intersection only if they are siblings, the partial function $f_{p}=\bigcup\left\{f_{s}: s \in \Gamma\right\} \bigcup \bigcup\left\{f_{t, u}: t, u\right.$ are siblings in $\left.\Theta\right\}$ is well-defined. Every total extension of $f_{p}$ is in $B_{X} \cap \bar{B}_{Y} \cap \bigcap_{s \in S} K_{s}$.

Theorem 3.5. The Cohen algebra embeds into r.o. $\left(\mathbf{P}_{A}\right)$ as a complete subalgebra.

Proof. We construct a countable, atomless, regular subalgebra $\mathbf{C}$ of r.o. $\left(\mathbf{P}_{A}\right)$. 
Construction of $\mathbf{C}$. Choose an infinite branch of $T$ and call it $\beta_{0} . \forall n<\omega$, let $t_{n}$ be the unique element in $\beta_{0} \cap \operatorname{Lev}(n)$, and choose one $s_{n+1} \in T \backslash \beta_{0}$ such that $s_{n+1}$ and $t_{n+1}$ are siblings. $\forall 0<n<\omega$, let $\beta_{n}$ be an infinite branch in $T$ which contains $s_{n}$. For $0<m<n, \beta_{m} \cap \beta_{n}=\left\{t_{0}, \ldots, t_{m-1}\right\}$. Define the following sets:

$$
T_{\mathbf{C}}=\left\{t \in T: t \in \bigcup_{n<\omega} \beta_{n} \text { or } t \text { is a sibling of some } s \in \bigcup_{n<\omega} \beta_{n}\right\},
$$

$$
N_{\mathbf{C}}=\bigcup T_{\mathbf{C}}=\left\{k<\omega: \exists l<\omega \text { such that }\{k, l\} \in T_{\mathbf{C}}\right\} .
$$

$T_{\mathbf{C}} \subseteq T$ and $N_{\mathbf{C}} \subseteq \omega$. Let $\mathcal{B} r_{\mathbf{C}}$ denote the set of all branches (finite and infinite) of $T_{\mathbf{C}} \cdot \mathcal{B} r_{\mathbf{C}}$ is countable, since $T_{\mathbf{C}}$ has only countably many infinite branches. Let

$$
\mathcal{C}=\left\{B_{X} \cap \bar{B}_{Y} \cap \bigcap_{\sigma \in \Sigma} A_{\sigma} \in \mathbf{P}_{A}: X, Y \in\left[N_{\mathbf{C}}\right]^{<\omega} \text { and } \Sigma \in\left[\mathcal{B} r_{\mathbf{C}}\right]^{<\omega}\right\} .
$$

Let

$$
\mathbf{C}=\langle\{e(p): p \in \mathcal{C}\}\rangle,
$$

the subalgebra of r.o. $\left(\mathbf{P}_{A}\right)$ generated by the set $\{e(p): p \in \mathcal{C}\}$. Note that $|\mathbf{C}|=\omega$, since $N_{\mathbf{C}}$ and $\mathcal{B} r_{\mathbf{C}}$ are countable.

Remark 3.6. The idea behind the choice of $T_{\mathbf{C}}$ and $N_{\mathbf{C}}$ is as follows: $T_{\mathbf{C}}$ was chosen so that for any finite set of branches $\Sigma \subseteq \mathcal{B} r_{\mathbf{C}}, \bigcap_{\sigma \in \Sigma} A_{\sigma} \neq \emptyset$. However, the subalgebra generated by $\left\{e\left(\bigcap_{\sigma \in \Sigma} A_{\sigma}\right): \Sigma \in\left[\mathcal{B} r_{\mathbf{C}}\right]^{<\omega}\right\}$ is not a regular subalgebra of r.o. $\left(\mathbf{P}_{A}\right)$. To enlarge it to a regular subalgebra, we chose $N_{\mathbf{C}}$ so that we can tell exactly how elements of the form $\bigcap_{\sigma \in \Sigma} A_{\sigma}$, $\Sigma \in\left[\mathcal{B} r_{\mathbf{C}}\right]^{<\omega}$, interact with members of $\mathbf{P}_{A}$. This allows us to find for each $p \in \mathbf{P}_{A}$ a minimal cover for $e(p)$ in $\mathbf{C}$, and thus ensure that $\mathbf{C}$ is a regular subalgebra of r.o. $\left(\mathbf{P}_{A}\right)$.

The following are two simple facts which we shall use without mention in subsequent proofs:

Fact 3.7. $\forall x \in \omega,-e\left(B_{\{x\}}\right)=e\left(\bar{B}_{\{x\}}\right)$.

Fact 3.8. $\forall p, q \in \mathbf{P}_{A}, e(p) \wedge e(q)=e(p \cap q)$.

Proposition 3.9. $\{e(p): p \in \mathcal{C}\}$ is dense in $\mathbf{C}^{+}$.

Proof. Every element of $\mathbf{C}$ is a finite disjunction of elements of the form

$$
e\left(B_{X} \cap \bar{B}_{Y} \cap \bigcap_{\sigma \in \Sigma} A_{\sigma}\right) \wedge \bigwedge_{\gamma \in \Gamma}-e\left(A_{\gamma}\right)
$$


where $X, Y \in\left[N_{\mathbf{C}}\right]^{<\omega}$ and $\Sigma, \Gamma \in\left[\mathcal{B} r_{\mathbf{C}}\right]^{<\omega}$. Let $c \in \mathbf{C}^{+}$be of the form (3.9), and let $p \in \mathbf{P}_{A}$ be such that $e(p) \leq c$. For each $\gamma \in \Gamma, e(p) \wedge e\left(A_{\gamma}\right)=\mathbf{0} \Longrightarrow$ $p \subseteq \bigcup_{s \in \gamma}\left(B_{s} \cup \bar{B}_{s}\right)$. Fix $f \in p$. For each $\gamma \in \Gamma$ choose an $s_{\gamma} \in \Gamma$ for which $f \in\left(B_{s_{\gamma}} \cup \bar{B}_{s_{\gamma}}\right)$. Define $\Gamma^{\prime}=\left\{\gamma \in \Gamma: f \in B_{s_{\gamma}}\right\}$ and $\Gamma^{\prime \prime}=\left\{\gamma \in \Gamma: f \in \bar{B}_{s_{\gamma}}\right\}$. Let

$$
q=B_{X \cup\left(\bigcup\left\{s_{\gamma}: \gamma \in \Gamma^{\prime}\right\}\right)} \cap \bar{B}_{Y \cup\left(\bigcup\left\{s_{\gamma}: \gamma \in \Gamma^{\prime \prime}\right\}\right)} \cap \bigcap_{\sigma \in \Sigma} A_{\sigma} .
$$

$f \in q$, so $q \neq \emptyset$. By its construction, $q \in \mathcal{C}$. Furthermore, $\forall \gamma \in \Gamma^{\prime}$, $e\left(B_{s_{\gamma}}\right) \leq-e\left(A_{\gamma}\right) ; \forall \gamma \in \Gamma^{\prime \prime}, e\left(\bar{B}_{s_{\gamma}}\right) \leq-e\left(A_{\gamma}\right) ;$ and $q \subseteq B_{X} \cap \bar{B}_{Y} \cap \bigcap_{\sigma \in \Sigma} A_{\sigma}$. Hence, $e(q) \leq c$.

\section{Proposition 3.10. $\mathrm{C}$ is atomless.}

Proof. It suffices to show that $\{e(p): p \in \mathcal{C}\}$ is atomless. Suppose $p \in \mathcal{C}$ and $B_{X} \cap \bar{B}_{Y} \cap \bigcap_{s \in S} K_{s}$ is the normal form of $p$. Choose some $z \in N_{\mathbf{C}} \backslash(X \cup Y)$ such that $z$ is neither in any member of $S$ nor in any sibling of any member of $S$. Then $B_{X \cup\{z\}} \cap \bar{B}_{Y} \cap \bigcap_{s \in S} K_{s}$ is the normal form of $p \cap B_{\{z\}}$; so by Lemma 3.4, $p \cap B_{\{z\}} \neq \emptyset$. Hence, $p \cap B_{\{z\}} \in \mathcal{C}$.

To see that $e\left(p \cap B_{\{z\}}\right)<e(p)$, note that $-e\left(B_{\{z\}}\right) \wedge e(p)=e\left(B_{X} \cap\right.$ $\left.\bar{B}_{Y \cup\{z\}} \cap \bigcap_{s \in S} K_{s}\right)$. This is strictly greater than $\mathbf{0}$, by Lemma 3.4, since $B_{X} \cap \bar{B}_{Y \cup\{z\}} \cap \bigcap_{s \in S} K_{s}$ is in the normal form.

We now show that $\mathbf{C}$ satisfies the conditions of Lemma 1.12.

Proposition 3.11. $\forall p \in \mathbf{P}_{A}, \exists c_{p} \in \mathbf{C}^{+}$such that $\forall c \in \mathbf{C}$, if $c_{p} \wedge c \neq \mathbf{0}$, then $e(p) \wedge c \neq \mathbf{0}$.

Proof. Let $p=B_{X} \cap \bar{B}_{Y} \cap \bigcap_{s \in S} K_{s} \in \mathbf{P}_{A} \cdot p \neq \emptyset$, so by Lemma 3.4, (L1 $p$ )-(L4 $p$ ) hold. Let $X^{\prime}=X \cap N_{\mathbf{C}}, Y^{\prime}=Y \cap N_{\mathbf{C}}, S^{\prime}=S \cap T_{\mathbf{C}}$, and $q=B_{X^{\prime}} \cap \bar{B}_{Y^{\prime}} \cap \bigcap_{s^{\prime} \in S^{\prime}} K_{s^{\prime}} . q \supseteq p$, and $q \in \mathcal{C}$ since $S^{\prime}$ is a finite union of branches in $T_{\mathbf{C}}$. Let $c_{p}=e(q) . \quad c_{p} \in \mathbf{C}^{+}$and $c_{p} \geq e(p)>\mathbf{0} . \quad\left(c_{p}\right.$ is actually the minimal cover for $e(p)$ in $\mathbf{C}$.) It suffices to show that $\forall r \in \mathcal{C}$, if $c_{p} \wedge e(r)>\mathbf{0}$, then $e(p) \wedge e(r)>\mathbf{0}$.

Suppose $r=B_{U} \cap \bar{B}_{V} \cap \bigcap_{w \in W} K_{w} \in \mathcal{C}$ and $c_{p} \wedge e(r)>\mathbf{0}$. Then

$$
q \cap r=B_{\left(X^{\prime} \cup U\right)} \cap \bar{B}_{\left(Y^{\prime} \cup V\right)} \cap \bigcap_{t \in S^{\prime} \cup W} K_{t} \neq \emptyset .
$$

Thus, Lemma 3.4 implies
$(\mathrm{L} 1 q \cap r) \quad\left(X^{\prime} \cup U\right) \cap\left(Y^{\prime} \cup V\right)=\emptyset$;
$(\mathrm{L} 2 q \cap r) \quad \forall s \in S^{\prime} \cup W, \quad s \nsubseteq X^{\prime} \cup U$ and $s \nsubseteq Y^{\prime} \cup V$;
(L3 $q \cap r) \quad S^{\prime} \cup W$ has no triplets;
$(\mathrm{L} 4 q \cap r) \quad s, t$ are siblings in $S^{\prime} \cup W \Longrightarrow\left((s \triangle t) \cap\left(X^{\prime} \cup U\right)=\emptyset\right.$ or $\left.(s \triangle t) \cap\left(Y^{\prime} \cup V\right)=\emptyset\right)$. 
It suffices to show that $p \cap r \neq \emptyset$. First, note that

$$
p \cap r=B_{\left(\left(X \backslash X^{\prime}\right) \cup X^{\prime} \cup U\right)} \cap \bar{B}_{\left(\left(Y \backslash Y^{\prime}\right) \cup Y^{\prime} \cup V\right)} \cap \bigcap_{s \in\left(S \backslash S^{\prime}\right) \cup S^{\prime} \cup W} K_{s},
$$

where $\left(X \backslash X^{\prime}\right) \cap N_{\mathbf{C}}=\left(Y \backslash Y^{\prime}\right) \cap N_{\mathbf{C}}=\emptyset ; X^{\prime} \cup U, Y^{\prime} \cup V \subseteq N_{\mathbf{C}} ;\left(S \backslash S^{\prime}\right) \cap T_{\mathbf{C}}=$ $\emptyset$; and $S^{\prime} \cup W \subseteq T_{\mathbf{C}}$.

Claim. (L1 $p \cap r)-(\mathrm{L} 4 p \cap r)$ hold.

(L1 $p \cap r)$ and (L2 $p \cap r)$ follow naturally from (L1 $p$ ), (L1 $q \cap r)$, (L2 $p$ ) and (L2 $q \cap r)$. $S^{\prime} \cup W$ and $S \backslash S^{\prime}$ each contain no triplets, by (L3 $q \cap r$ ) and (L3 $p$ ), respectively. $S \backslash S^{\prime} \subseteq T \backslash T_{\mathbf{C}}$ and $S^{\prime} \cup W \subseteq T_{\mathbf{C}}$ imply $S \backslash S^{\prime}$ and $S^{\prime} \cup W$ have no common siblings. Hence, $S \cup W$ contains no triplets, so (L3 $p \cap r)$ holds.

Suppose $s, t$ are siblings in $S \cup W$. Then either $s, t \in S \backslash S^{\prime}$ or else $s, t \in$ $S^{\prime} \cup W$. Suppose $s, t \in S \backslash S^{\prime}$. Then $(s \cup t) \cap N_{\mathbf{C}}=\emptyset$, so $(s \triangle t) \cap\left(X^{\prime} \cup\right.$ $\left.Y^{\prime} \cup U \cup V\right)=\emptyset$. Further, (L4p) implies either $(s \triangle t) \cap X=\emptyset$ or else $(s \triangle t) \cap Y=\emptyset$. On the other hand, if $s, t \in S^{\prime} \cup W$, then $s \cup t \subseteq N_{\mathbf{C}}$, so $(s \cup t) \cap\left(\left(X \backslash X^{\prime}\right) \cup\left(Y \backslash Y^{\prime}\right)\right)=\emptyset$. By $(\mathrm{L} 4 q \cap r)$, either $(s \triangle t) \cap\left(X^{\prime} \cup U\right)=\emptyset$ or else $(s \triangle t) \cap\left(Y^{\prime} \cup V\right)=\emptyset$. Thus, in both cases, either $(s \triangle t) \cap(X \cup U)=\emptyset$ or else $(s \triangle t) \cap(Y \cup V)=\emptyset$. Hence, (L4 $p \cap r)$ holds.

By Lemma 3.4, $p \cap r \neq \emptyset$. Thus, $e(p) \wedge e(r)>\mathbf{0}$.

By Proposition 3.11 and Lemmas 1.12 and 1.15, r.o. $\left(\mathbf{C}^{+}\right)$is a complete subalgebra of r.o. $\left(\mathbf{P}_{A}\right)$. This completes our construction of a complete embedding of the Cohen algebra into the Argyros algebra.

Remark 3.12. We are very grateful to the referee for suggesting that we investigate Argyros' other variants of this example in which stronger chain conditions hold, to see whether the Cohen algebra completely embeds in these. We have found that it does.

Theorem 3.13 (Argyros, $[\mathbf{C N}]$, p. 156.). For each $2 \leq m<\omega$, there is a family of atomless, separative partial orderings $\mathbf{P}_{m}$ such that each $\mathbf{P}_{m}$ satisfies the $\sigma$-bounded c.c. and property $K_{m}, \operatorname{CUP}\left(\mathbf{P}_{m}\right)$ fails, and, assuming $\mathrm{CH}$, property $K_{m+1}$ fails.

Our preceding construction can be easily modified to completely embed the Cohen algebra into each r.o. $\left(\mathbf{P}_{m}\right)$.

Theorem 3.14. For each $2 \leq m<\omega$, the Cohen algebra embeds as a complete subalgebra into r.o. $\left(\mathbf{P}_{m}\right)$.

The modification is as follows: Let $m$ be given. Argyros constructed a family of trees $T$ such that each $T$ is an $(m+1)$-branching tree of height $\omega$ with the following properties: For each node $\sigma \in T$, there is a finite subset, $\operatorname{dom}(\sigma) \subseteq \omega$, such that $\sigma$ is a set of functions from $\operatorname{dom}(\sigma)$ to 2 . All 
siblings have the same domain, and any two nodes which are not siblings have disjoint domains. Any $m$ siblings have nonempty intersection, but the intersection of $(m+1)$-many siblings is empty. (See $[\mathbf{C N}]$ p. 156 for the precise definition of $T_{m}$.) For $\sigma \in T$, let $A_{\sigma}=\left\{f \in 2^{\omega}: f\lceil\operatorname{dom}(\sigma) \in \sigma\}\right.$. $\mathbf{P}_{m}=\left\{B_{X} \cap \bar{B}_{Y} \cap \bigcap_{\sigma \in \Sigma} A_{\sigma}: X, Y \in[\omega]^{<\omega}\right.$ and $\Sigma$ is a finite set of branches in $T\} \backslash\{\emptyset\}$, partially ordered by inclusion.

To construct a countable, atomless, regular subalgebra of r.o. $\left(\mathbf{P}_{m}\right)$, take, as before, $\omega$-many infinite branches $\beta_{i}$ in $T$ such that for each node $\sigma \in$ $\bigcup_{i<\omega} \beta_{i}, \sigma$ has at most one sibling in $\bigcup_{i<\omega} \beta_{i}$. Let $T_{\mathbf{C}}$ be the subtree of $T$ consisting of $\bigcup_{i<\omega} \beta_{i}$ and all siblings of nodes in $\bigcup_{i<\omega} \beta_{i}$. Let $N_{\mathbf{C}}=$ $\bigcup\left\{\operatorname{dom}(\sigma): \sigma \in T_{\mathbf{C}}\right\}$. Let $\mathbf{C}$ be the subalgebra of r.o. $\left(\mathbf{P}_{m}\right)$ generated by the elements of the form $B_{X} \cap \bar{B}_{Y} \cap \bigcap_{\sigma \in \Sigma} A_{\sigma}$ such that $X, Y$ are finite subsets of $N_{\mathbf{C}}$ and $\Sigma$ is a finite set of branches in $T_{\mathbf{C}}$. The proof that this subalgebra is atomless and regular proceeds as before.

\section{Atomless and non-atomless Gaifman algebras.}

Gaifman constructed a family of Boolean algebras $\mathbf{B}_{G}$ as follows $[\mathbf{G a}]$ : Let $\operatorname{Clop}\left(2^{(0,1)}\right)$ denote the clopen subsets of $2^{(0,1)}$. For $X, Y \in[(0,1)]^{<\omega}$, let

$$
\begin{aligned}
& B_{X}=\left\{f \in 2^{(0,1)}: \forall x \in X, f(x)=1\right\}, \\
& \bar{B}_{Y}=\left\{f \in 2^{(0,1)}: \forall y \in Y, f(y)=0\right\} .
\end{aligned}
$$

Let $\left\{T_{i}: 2 \leq i<\omega\right\}$ be an enumeration of the open subintervals of $(0,1)$ with rational endpoints. For each $2 \leq i<\omega$, choose $i^{2}$-many disjoint, open subintervals of $T_{i}$ and label them $T_{i 1}, T_{i 2}, \ldots, T_{i i^{2}}$. We let $T_{i 0}=$ $(0,1) \backslash \bigcup_{1 \leq j \leq i^{2}} T_{i j}$, so that $\left\{T_{i j}: 0 \leq j \leq i^{2}\right\}$ is a partition of $(0,1)$. Let $I$ be the set of those elements of $\operatorname{Clop}\left(2^{(0,1)}\right)$ of the form $B_{X} \cap \bar{B}_{Y}$ such that for some $2 \leq i<\omega, X$ intersects at least $i$-many of the open intervals $T_{i 1}, \ldots, T_{i i^{2}}$. Let $\mathcal{I}$ be the ideal generated by $I$ in $\operatorname{Clop}\left(2^{(0,1)}\right)$. The Gaifman algebra is the quotient algebra

$$
\mathbf{B}_{G}=\operatorname{Clop}\left(2^{(0,1)}\right) / \mathcal{I}
$$

For $c \in \operatorname{Clop}\left(2^{(0,1)}\right)$ we shall denote the equivalence class of $c$ in $\mathbf{B}_{G}$ by $[c]$.

Notice that the set $\left\{\left[B_{X} \cap \bar{B}_{Y}\right]: X, Y \in[(0,1)]^{<\omega}\right.$ and $\left.B_{X} \cap \bar{B}_{Y} \notin \mathcal{I}\right\}$ is dense in $\mathbf{B}_{G}{ }^{+}$. We will use this fact implicitly in this and the next section.

Theorem 4.1 (Gaifman, $[\mathbf{G a}]$ ). $\mathbf{B}_{G}$ satisfies the $\sigma$-bounded c.c., but does not satisfy $\operatorname{CUP}\left(\mathbf{B}_{G}{ }^{+}\right)$.

By Kelley's Theorem 1.9, $\mathbf{B}_{G}$ is not measurable.

Depending on how the $T_{i}$ 's and $T_{i j}$ 's are chosen, $\mathbf{B}_{G}$ may or may not have atoms. To show this, we will use Lemmas 4.2 and 4.4 and Fact 4.3. 
Lemma 4.2 (Gaifman, [Ga]). For each $B_{X} \cap \bar{B}_{Y} \in \operatorname{Clop}\left(2^{(0,1)}\right)$ with $X \cap$ $Y=\emptyset, B_{X} \cap \bar{B}_{Y} \in \mathcal{I}$ iff $B_{X} \in \mathcal{I}$.

The next fact follows easily from Lemma 4.2.

Fact 4.3. Suppose $\left[B_{U} \cap \bar{B}_{V}\right] \leq\left[B_{X} \cap \bar{B}_{Y}\right]$ in $\mathbf{B}_{G}{ }^{+}$. Then $X \subseteq U$ and $(V \cup Y) \cap U=\emptyset$. If in addition $U \backslash X \neq \emptyset$, then $\left[B_{U} \cap \bar{B}_{V}\right]<\left[B_{X} \cap \bar{B}_{Y}\right]$.

The following lemma gives necessary and sufficient conditions for an element of $\mathbf{B}_{G}{ }^{+}$to be an atom:

Lemma 4.4. $b \in \mathbf{B}_{G}^{+}$is an atom $\Longleftrightarrow$ for some $X, Y \in[(0,1)]<\omega, b=$ $\left[B_{X} \cap \bar{B}_{Y}\right]>[\mathbf{0}]$ and for each $x \in(0,1) \backslash(X \cup Y), B_{X} \cap B_{\{x\}} \in \mathcal{I}$.

Proof. Suppose $b$ is an atom. Then there must exist $X, Y \in[(0,1)]^{<\omega}$ such that $b=\left[B_{X} \cap \bar{B}_{Y}\right]$. If $\exists x \in(0,1) \backslash(X \cup Y)$ such that $B_{X} \cap B_{\{x\}} \notin \mathcal{I}$, then by Lemma 4.2 and Fact 4.3, $[\mathbf{0}]<\left[B_{X} \cap B_{\{x\}} \cap \bar{B}_{Y}\right]<\left[B_{X} \cap \bar{B}_{Y}\right]$. Contradiction.

Conversely, suppose $b=\left[B_{X} \cap \bar{B}_{Y}\right]>[\mathbf{0}]$ and $\forall x \in(0,1) \backslash(X \cup Y)$, $B_{X} \cap B_{\{x\}} \in \mathcal{I}$. Suppose also that $\left[B_{U} \cap \bar{B}_{V}\right]$ is such that $\left[B_{U} \cap \bar{B}_{V}\right] \wedge$ $\left[B_{X} \cap \bar{B}_{Y}\right]>[\mathbf{0}]$. Then $X \cap V=\emptyset$, so $\forall v \in V \backslash Y, B_{X} \cap B_{\{v\}} \in \mathcal{I}$. Thus, $\left[\bar{B}_{V \backslash Y}\right] \geq\left[B_{X}\right]$. Furthermore, $B_{U} \cap B_{X} \notin \mathcal{I}$, so $U$ must be contained in $X$. Therefore, $\left[B_{U} \cap \bar{B}_{V}\right] \geq\left[B_{X} \cap \bar{B}_{Y}\right]$. Hence, $b$ is an atom.

Depending on the intervals $T_{i}, T_{i j}$ used in the construction, a Gaifman algebra may have atoms. The following is an atom in many Gaifman algebras:

Example 4.5 (Some Gaifman algebras have atoms). Let $T_{2}=(0,1), T_{3}=$ $\left(0, \frac{31}{32}\right)$, and $T_{4}=\left(0, \frac{59}{64}\right)$, and choose the following $T_{i j}$ 's in these $T_{i}$ 's:

$$
\begin{aligned}
\left\{T_{2,1}, \ldots, T_{2,4}\right\}=\{ & \left.\left(0, \frac{3}{4}\right),\left(\frac{3}{4}, \frac{7}{8}\right),\left(\frac{7}{8}, \frac{15}{16}\right),\left(\frac{15}{16}, 1\right)\right\} \\
\left\{T_{3,1}, \ldots, T_{3,9}\right\}=\{ & \left(0, \frac{1}{16}\right),\left(\frac{1}{16}, \frac{3}{16}\right),\left(\frac{3}{16}, \frac{5}{16}\right),\left(\frac{5}{16}, \frac{7}{16}\right),\left(\frac{7}{16}, \frac{9}{16}\right),\left(\frac{9}{16}, \frac{11}{16}\right), \\
& \left.\left(\frac{11}{16}, \frac{13}{16}\right),\left(\frac{13}{16}, \frac{29}{32}\right),\left(\frac{29}{32}, \frac{31}{32}\right)\right\} \\
\left\{T_{4,1}, \ldots, T_{4,16}\right\}=\{( & \left(0, \frac{1}{32}\right),\left(\frac{1}{32}, \frac{3}{32}\right),\left(\frac{3}{32}, \frac{5}{32}\right),\left(\frac{5}{32}, \frac{7}{32}\right),\left(\frac{7}{32}, \frac{9}{32}\right),\left(\frac{9}{32}, \frac{11}{32}\right), \\
& \left(\frac{11}{32}, \frac{13}{32}\right),\left(\frac{13}{32}, \frac{15}{32}\right),\left(\frac{15}{32}, \frac{17}{32}\right),\left(\frac{17}{32}, \frac{19}{32}\right),\left(\frac{19}{32}, \frac{21}{32}\right), \\
& \left.\left(\frac{21}{32}, \frac{23}{32}\right),\left(\frac{23}{32}, \frac{25}{32}\right),\left(\frac{25}{32}, \frac{27}{32}\right),\left(\frac{27}{32}, \frac{57}{64}\right),\left(\frac{57}{64}, \frac{59}{64}\right)\right\} .
\end{aligned}
$$

Let

$$
X=\left\{\frac{1}{32}, \frac{3}{32}, \frac{5}{32}, \frac{10}{32}, \frac{14}{32}, \frac{18}{32}\right\}
$$


For many choices of $T_{5}, T_{6}, T_{7}$ 's, $\left[B_{X}\right]$ is an atom in the corresponding Gaifman algebra. For instance, if $T_{5}, T_{6}, T_{7} \subseteq\left(\frac{31}{32}, 1\right)$, then no matter how the $T_{i}$ for $i \geq 8$ and $T_{i j}$ for $i \geq 5$ are chosen, $\left[B_{X}\right]>[\mathbf{0}]$. Furthermore, for any $x \in(0,1) \backslash X, B_{X \cup\{x\}} \in \mathcal{I}$, since $x$ must lie in at least one of the $T_{2, j}$ 's, $T_{3, j}$ 's, or $T_{4, j}$ 's $(j \geq 1)$ which $X$ does not intersect. Thus, by Lemma 4.4 , $\left[B_{X}\right]$ is an atom.

Remark 4.6. Every Gaifman algebra has at most countably many atoms, since the c.c.c. holds.

Every Gaifman algebra has a large atomless part. Let

$$
E=\bigcup_{2 \leq i<\omega}\left(T_{i 0} \backslash \operatorname{int}\left(T_{i 0}\right)\right) .
$$

$E$ is the set of all endpoints of the intervals $T_{i j}, 2 \leq i<\omega, 1 \leq j \leq i^{2}$.

Lemma 4.7. If $z \in(0,1) \backslash E$, then there are no atoms in $\mathbf{B}_{G}$ below $\left[B_{\{z\}}\right]$.

Proof. Suppose $z \in(0,1) \backslash E$ and $[\mathbf{0}]<\left[B_{U} \cap \bar{B}_{V}\right] \leq\left[B_{\{z\}}\right]$. Then $z \in U$, by Fact 4.3 . For each $i \geq 2$ let $j(i) \leq i^{2}$ be such that $z \in \operatorname{int}\left(T_{i, j(i)}\right)$, and let $N=|U|+2$. Then $\operatorname{int}\left(\bigcap_{2 \leq i \leq N} T_{i, j(i)}\right) \neq \emptyset$, so choose a $z^{\prime} \in$ $\operatorname{int}\left(\bigcap_{2 \leq i \leq N} T_{i, j(i)}\right) \backslash(U \cup V) . \quad \forall 2 \leq i \leq N, z, z^{\prime} \in T_{i, j(i)}$, so $U \cup\left\{z^{\prime}\right\}$ has nonempty intersection with at most $(i-1)$-many $T_{i j}$ 's. Thus, $[\mathbf{0}]<$ $\left[B_{U \cup\left\{z^{\prime}\right\}} \cap \bar{B}_{V}\right]$. Fact 4.3 implies $\left[B_{U \cup\left\{z^{\prime}\right\}} \cap \bar{B}_{V}\right]<\left[B_{U} \cap \bar{B}_{V}\right]$, since $z^{\prime} \notin U$. Hence, $\left[B_{U} \cap \bar{B}_{V}\right]$ is not an atom.

\section{Remark 4.8.}

1. By Lemma 4.7, $\left[B_{X} \cap \bar{B}_{Y}\right]$ is an atom only if $X \subseteq E$. Moreover, if $\left[B_{X} \cap \bar{B}_{Y}\right]$ is an atom, then $X \subseteq \bigcup_{2 \leq i \leq|X|+1}\left(T_{i 0} \backslash \operatorname{int}\left(T_{i 0}\right)\right)$.

2. Atomless Gaifman algebras do exist. For example, if the $T_{i j}$ 's are nested so that $\left(i<k, 0 \leq j \leq i^{2}, 1 \leq l \leq k^{2}\right.$, and $\left.T_{i j} \cap T_{k l} \neq \emptyset\right) \Longrightarrow$ $T_{k l} \subseteq T_{i j}$, then the resulting Gaifman algebra is atomless.

\section{The Cohen algebra completely embeds into the atomless part of each Gaifman algebra.}

In this section, we work in the atomless part of Gaifman algebras. Let $\mathbf{B}_{G}$ be a Gaifman algebra. We identify $\mathbf{B}_{G}{ }^{+}$with its image under the canonical dense embedding of $\mathbf{B}_{G}{ }^{+}$into r.o. $\left(\mathbf{B}_{G}{ }^{+}\right)$and work in r.o. $\left(\mathbf{B}_{G}{ }^{+}\right)$. Let

$$
a=[\mathbf{1}] \backslash\left(\bigvee\left\{b \in \text { r.o. }\left(\mathbf{B}_{G}^{+}\right): b \text { is an atom }\right\}\right)
$$

in r.o. $\left(\mathbf{B}_{G}^{+}\right)$, and define

$$
\mathbf{A}_{G}=\operatorname{r.o.}\left(\mathbf{B}_{G}^{+}\right) \uparrow a .
$$


$\mathbf{A}_{G}$ is the atomless part of r.o. $\left(\mathbf{B}_{G}^{+}\right)$. Since r.o. $\left(\mathbf{B}_{G}^{+}\right)$satisfies the $\sigma$ bounded c.c., $\mathbf{A}_{G}$ also satisfies the $\sigma$-bounded c.c. Since $\operatorname{CUP}\left(\right.$ r.o. $\left.\left(\mathbf{B}_{G}{ }^{+}\right)^{+}\right)$ fails and r.o. $\left(\mathbf{B}_{G}{ }^{+}\right)$has at most countably many atoms, $\operatorname{CUP}\left(\mathbf{A}_{G}{ }^{+}\right)$must fail. Thus, by Kelley's Theorem 1.9, $\mathbf{A}_{G}$ is non-measurable.

To avoid confusing notation between elements of r.o. $\left(\mathbf{B}_{G}{ }^{+}\right)$and $\mathbf{A}_{G}$, we will hold to the convention that $\left[B_{X} \cap \bar{B}_{Y}\right]$ always refers to an element of r.o. $\left(\mathbf{B}_{G}{ }^{+}\right)$. We will often use without mention the fact that $\left\{\left[B_{X} \cap \bar{B}_{Y}\right] \in\right.$ r.o. $\left.\left(\mathbf{B}_{G}{ }^{+}\right):[\mathbf{0}]<\left[B_{X} \cap \bar{B}_{Y}\right] \leq a\right\}$ is a dense subset of $\mathbf{A}_{G}{ }^{+}$.

Lemma 5.1. If $F \subseteq(0,1)$ is finite and $\left[B_{X} \cap \bar{B}_{Y}\right] \in \mathbf{A}_{G}{ }^{+}$, then $\exists z \in$ $(0,1) \backslash(F \cup X \cup Y)$ such that $[\mathbf{0}]<\left[B_{X \cup\{z\}} \cap \bar{B}_{Y}\right]$.

Proof.

Claim. $\forall\left[B_{X} \cap \bar{B}_{Y}\right] \in \mathbf{A}_{G}{ }^{+}, \exists z \in(0,1) \backslash(X \cup Y)$ for which $[\mathbf{0}]<\left[B_{X \cup\{z\}} \cap\right.$ $\left.\bar{B}_{Y}\right]$. Suppose $\left[B_{X} \cap \bar{B}_{Y}\right] \in \mathbf{A}_{G}{ }^{+}$. Since $\mathbf{A}_{G}$ is atomless, $\exists\left[B_{U} \cap \bar{B}_{V}\right] \in \mathbf{A}_{G}{ }^{+}$ such that $\left[B_{U} \cap \bar{B}_{V}\right]<\left[B_{X} \cap \bar{B}_{Y}\right]$. By Fact $4.3, U \supseteq X$, so $\left[B_{U} \cap \bar{B}_{V}\right]=\left[B_{U} \cap\right.$ $\left.\bar{B}_{V \cup Y}\right]$. Since $\mathbf{A}_{G}$ is atomless, Lemma 4.4 implies $\exists z \in(0,1) \backslash(U \cup V \cup Y)$ such that $B_{U \cup\{z\}} \notin \mathcal{I}$. Lemma 4.2 implies $\left[B_{U \cup\{z\}} \cap \bar{B}_{V \cup Y}\right]>[\mathbf{0}]$. Hence, the Claim holds.

Let $n=|F|$. By the Claim, we can inductively choose a sequence of distinct elements $z_{0}, \ldots, z_{n} \in(0,1) \backslash(X \cup Y)$ such that $[\mathbf{0}]<\left[B_{X \cup\left\{z_{0}, \ldots, z_{n}\right\}} \cap\right.$ $\left.\bar{B}_{Y}\right]$. Thus, there is some $0 \leq i \leq n$ such that $z_{i} \notin F \cup X \cup Y$ and $[\mathbf{0}]<\left[B_{X \cup\left\{z_{i}\right\}} \cap \bar{B}_{Y}\right]$.

Theorem 5.2. The Cohen algebra embeds as a complete subalgebra into $\mathbf{A}_{G}$.

Proof. We construct a countable, atomless, regular subalgebra $\mathbf{C}$ of $\mathbf{A}_{G}$. Recall that $E$ denotes the set of all endpoints of the intervals $T_{i j}, 2 \leq i<$ $\omega, 1 \leq j \leq i^{2}$ (see (4.4)). Our construction uses two types of sets: $F_{i}$ 's which keep track of elements of $E$, and $X_{i}$ 's which keep track of elements of $(0,1) \backslash E$. We start by constructing the $F_{i}$ 's recursively.

Construction of $\mathbf{C}$. Let $E_{2}=T_{20} \backslash \operatorname{int}\left(T_{20}\right)$, the endpoints of the open intervals $T_{21}, T_{22}, T_{23}, T_{24}$. Let

$$
F_{2}=E_{2} .
$$

Let $E_{3}=T_{30} \backslash\left(\operatorname{int}\left(T_{30}\right) \cup F_{2}\right)$. Recall that $a$ is the complement of the supremum of the atoms in r.o. $\left(\mathbf{B}_{G}{ }^{+}\right)$(see (5.1)). $\forall F \subseteq F_{2}$ for which $\left[B_{F}\right] \wedge a>[\mathbf{0}]$, choose one $x_{F} \in(0,1) \backslash F_{2}$ such that $\left[B_{F \cup\left\{x_{F}\right\}}\right] \wedge a>[\mathbf{0}]$. This is possible by Lemma 5.1 and the fact that $\left\{\left[B_{X} \cap \bar{B}_{Y}\right] \in \operatorname{r.o.}\left(\mathbf{B}_{G}^{+}\right):\left[B_{X} \cap \bar{B}_{Y}\right] \leq a\right\}$ is dense in $\mathbf{A}_{G}{ }^{+}$. Let

$$
F_{3}=E_{3} \cup\left\{x_{F}: F \subseteq F_{2},\left[B_{F}\right] \wedge a>[\mathbf{0}]\right\} .
$$


Given $F_{2}, \ldots, F_{n}$, let $E_{n+1}=T_{n+1,0} \backslash\left(\operatorname{int}\left(T_{n+1,0}\right) \cup \bigcup_{2<i<n} F_{i}\right)$. Again, $\forall F \subseteq$ $\bigcup_{2 \leq i \leq n} F_{i}$ for which $\left[B_{F}\right] \wedge a>[\mathbf{0}]$, choose one $x_{F} \in(\overline{0}, \overline{1}) \backslash\left(\bigcup_{2 \leq i \leq n} F_{i}\right)$ such that $\left[B_{F \cup\left\{x_{F}\right\}}\right] \wedge a>[\mathbf{0}]$. Let

$$
F_{n+1}=E_{n+1} \cup\left\{x_{F}: F \subseteq \bigcup_{2 \leq i \leq n} F_{i},\left[B_{F}\right] \wedge a>[\mathbf{0}]\right\} .
$$

The sets $F_{i}$ are finite and have the following properties:

(F1) $E \subseteq \bigcup_{2 \leq i<\omega} F_{i}$;

(F2) $\forall 2 \leq i<j<\omega, F_{i} \cap F_{j}=\emptyset$;

(F3) $\forall 2 \leq j<\omega, \forall F \subseteq \bigcup_{2 \leq k<j} F_{k}$ such that $\left[B_{F}\right] \wedge a>[\mathbf{0}]$, there is an $x_{F} \in F_{j}$ for which $\left[B_{F \cup\left\{x_{F}\right\}}\right] \wedge a>[\mathbf{0}]$.

Taking the $F_{i}$ 's into consideration, we construct finite sets $X_{i}$ recursively. For $i \geq 2$, let

$$
J_{i}=\left\{s=\langle s(2), \ldots, s(i+1)\rangle \in \prod_{k=2}^{i+1}\left(k^{2}+1\right): \operatorname{int}\left(\bigcap_{k=2}^{i+1} T_{k, s(k)}\right) \neq \emptyset\right\} .
$$

$J_{i} \neq \emptyset$, since $\forall 2 \leq k \leq i^{2}+1,\left\{T_{k j}: j \leq k^{2}\right\}$ is a partition of $(0,1)$ into finitely many open intervals and a finite union of closed intervals. For each $s \in J_{i}$, choose one $x_{s} \in \operatorname{int}\left(\bigcap_{2 \leq k \leq i+1} T_{k, s(k)}\right) \backslash\left(\bigcup_{2 \leq k<\omega} F_{k} \cup \bigcup_{2 \leq k<i} X_{k}\right)$. Let

$$
X_{i}=\left\{x_{s}: s \in J_{i}\right\} \text {. }
$$

The sets $X_{i}, F_{i}$ have the following properties:

(XF1) $\left(\bigcup_{2 \leq i<\omega} X_{i}\right) \cap\left(\bigcup_{2 \leq j<\omega} F_{j}\right)=\emptyset$;

(XF2) $\forall 2 \leq i<j<\omega,\left(X_{i} \cup F_{i}\right) \cap\left(X_{j} \cup F_{j}\right)=\emptyset$.

For each $2 \leq i<\omega$, define (in r.o. $\left(\mathbf{B}_{G}{ }^{+}\right)$)

$$
c_{i}=\left(\bigvee_{x \in X_{i}}\left[B_{\{x\}}\right] \vee \bigvee_{f \in F_{i}}\left[B_{\{f\}}\right]\right) \wedge a \text {. }
$$

Note that by Lemma 4.7, for each $x \in \bigcup_{2 \leq i<\omega} X_{i},\left[B_{\{x\}}\right] \in \mathbf{A}_{G}$, since $x \notin E$. Hence, $c_{i}=\bigvee_{x \in X_{i}}\left[B_{\{x\}}\right] \vee\left(\bigvee_{f \in F_{i}}\left[B_{\left\{f_{i}\right\}}\right] \wedge a\right)$. Let

$$
\mathbf{C}=\left\langle\left\{c_{i}: 2 \leq i<\omega\right\}\right\rangle,
$$

the subalgebra of $\mathbf{A}_{G}$ generated by $\left\{c_{i}: 2 \leq i<\omega\right\}$. By our notational convention, the complement of $c_{i}$ in $\mathbf{A}_{G}$ will be written as $-c_{i} \wedge a$, where $-c_{i}$ denotes the complement of $c_{i}$ in r.o. $\left(\mathbf{B}_{G}{ }^{+}\right)$.

Proposition 5.3. The generators of $\mathbf{C}$ are independent. 
Proof. Suppose $K, L$ are finite, disjoint subsets of $\{2,3,4, \ldots\}$.

$$
\bigwedge_{k \in K} c_{k} \wedge \bigwedge_{l \in L}\left(-c_{l} \wedge a\right)=\bigwedge_{k \in K}\left(\bigvee_{u \in X_{k} \cup F_{k}}\left[B_{\{u\}}\right]\right) \wedge\left[\bar{B}_{\cup_{l \in L}\left(X_{l} \cup F_{l}\right)}\right] \wedge a .
$$

If $K=\emptyset$, the right-hand side of (5.10) becomes $\left[\bar{B}_{\cup_{l \in L}\left(X_{l} \cup F_{l}\right)}\right] \wedge a$, which is greater than $[\mathbf{0}]$. Suppose $K \neq \emptyset$. It suffices to find a sequence $\left\langle x_{k}: k \in\right.$

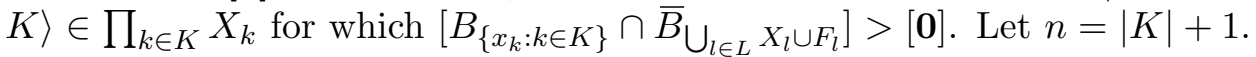
Order the elements of $K$ as $k_{2}<k_{3}<\cdots<k_{n}$. Fix an $x_{2} \in X_{k_{2}} . \forall 2 \leq i \leq$ $k_{n}+1$, let $s(i) \leq i^{2}$ be such that $x_{2} \in T_{i, s(i)}$. By (F1) and (XF1), $x_{2} \notin E$, so $x_{2} \in \operatorname{int}\left(\bigcap_{2 \leq i<k_{n}+1} T_{i, s(i)}\right)$. Thus, $\forall 3 \leq m \leq n,\left\langle s(2), s(3), \ldots, s\left(k_{m}+1\right)\right\rangle \in$ $J_{k_{m}}$, so choose $x_{m} \in X_{k_{m}} \cap \operatorname{int}\left(\bigcap_{2 \leq i \leq k_{m}+1} T_{i, s(i)}\right)$. Let $X=\left\{x_{m}: 2 \leq m \leq\right.$ $n\}$. For each $2 \leq i \leq n-1, k_{i} \geq i$, so $\left\{x_{2}, x_{i-1}, \ldots, x_{n}\right\}$ all lie in $T_{i, s(i)}$. Therefore, $X$ intersects at most $(i-3)$-many $T_{i j}$ 's. Since $|X| \leq|K|<n$, $B_{X} \notin \mathcal{I}$. By $(\mathrm{XF} 2)$ and Lemmas 4.2 and 4.7, $a \geq\left[B_{X} \cap \bar{B}_{\cup_{l \in L} X_{l} \cup F_{l}}\right]>$ $[\mathbf{0}]$.

By Proposition 5.3, $\mathbf{C}$ is atomless.

Proposition 5.4. For each $d \in \mathbf{A}_{G}{ }^{+}$, there exists a $c_{d} \in \mathbf{C}^{+}$such that whenever $c \in \mathbf{C}$ and $c \wedge c_{d} \neq[\mathbf{0}]$, then $c \wedge d \neq[\mathbf{0}]$.

Proof. It suffices to show the proposition for all $d \in \mathbf{A}_{G}{ }^{+}$of the form $\left[B_{X} \cap\right.$ $\left.\bar{B}_{Y}\right]$. Let $d=\left[B_{X} \cap \bar{B}_{Y}\right] \in \mathbf{A}_{G}{ }^{+}$. By (XF2), we can fix an $N \geq|X \cup Y|+1$ such that $(X \cup Y) \cap\left(\bigcup_{N<i<\omega}\left(X_{i} \cup F_{i}\right)\right)=\emptyset$. Define $I=\{2 \leq i \leq N$ : $\left.X \cap\left(X_{i} \cup F_{i}\right) \neq \emptyset\right\}$ and $J=\left\{2 \leq i \leq N: X \cap\left(X_{i} \cup F_{i}\right)=\emptyset\right\}$. Let

$$
c_{d}=\bigwedge_{i \in I} c_{i} \wedge \bigwedge_{j \in J}\left(-c_{j} \wedge a\right)
$$

$c_{d}>[\mathbf{0}]$ by Proposition 5.3. (Unlike in our constructions of complete embeddings of the Cohen algebra into the families of Galvin-Hajnal and Argyros algebras, $c_{d}$ is not necessarily a minimal cover for $d$ in $\mathbf{C}$.)

Suppose $c=\bigwedge_{k \in K} c_{k} \wedge \bigwedge_{l \in L}\left(-c_{l} \wedge a\right) \in \mathbf{C}^{+}$, where $K, L$ are finite subsets of $\{2,3, \ldots\}$, and $c_{d} \wedge c>[\mathbf{0}]$. Then by Proposition 5.3, $(I \cup K) \cap(J \cup L)=\emptyset$. Let $K^{\prime}=K \cap\{2, \ldots, N\}$ and $K^{\prime \prime}=K \backslash K^{\prime} . K \cap J=\emptyset$ implies $K^{\prime} \subseteq I$. Since $d \leq a$,

$$
c \wedge d=\bigwedge_{k \in K}\left(\bigvee_{u \in X_{k} \cup F_{k}}\left[B_{\{u\}}\right]\right) \wedge\left[B_{X} \cap \bar{B}_{Y \cup \cup_{l \in L}\left(X_{l} \cup F_{l}\right)}\right] .
$$

$\forall k \in K^{\prime},\left(X_{k} \cup F_{k}\right) \cap X \neq \emptyset$, since $K^{\prime} \subseteq I$. Thus, by Lemma 4.2, to show that $c \wedge d>[\mathbf{0}]$, it suffices to find a sequence $\left\langle u_{k}: k \in K^{\prime \prime}\right\rangle \in \prod_{k \in K^{\prime \prime}}\left(X_{k} \cup F_{k}\right)$ for which: 
(a) $\left[B_{\left\{u_{k}: k \in K^{\prime \prime}\right\} \cup X}\right]>[\mathbf{0}]$; and

(b) $\left(\left\{u_{k}: k \in K^{\prime \prime}\right\} \cup X\right) \cap\left(Y \cup \bigcup_{l \in L}\left(X_{l} \cup F_{l}\right)\right)=\emptyset$.

Let $m=\left|K^{\prime \prime}\right|-1$ and list the elements of $K^{\prime \prime}$ as $N+1 \leq k_{0}<k_{1}<\cdots<$ $k_{m}$. There are two cases for (a).

If $X \subseteq E$, then since $E \subseteq \bigcup_{2 \leq i<\omega} F_{i}$ and $X \cap\left(\bigcup_{N<i<\omega} F_{i}\right)=\emptyset$, it follows that $X \subseteq \bigcup_{k \in K^{\prime}} F_{k}$. [B $\left.B_{X}\right] \wedge a>[\mathbf{0}]$ and (F3) imply $\exists f_{k_{0}} \in F_{k_{0}}$ such that $\left[B_{X \cup\left\{f_{k_{0}}\right\}}\right] \wedge a>[\mathbf{0}]$. By induction using (F3), we obtain a sequence $\left\langle f_{k_{0}}, \ldots, f_{k_{m}}\right\rangle \in \prod_{0 \leq j \leq m} F_{k_{j}}$ for which $\left[B_{X \cup\left\{f_{k_{0}}, \ldots, f_{k_{m}}\right\}}\right] \wedge a>[\mathbf{0}]$. Thus, (a) holds for the sequence $\left\langle f_{k}: k \in K^{\prime \prime}\right\rangle$.

If $X \backslash E \neq \emptyset$, then fix an element $x \in X \backslash E$. Let $s \in \prod_{2 \leq i \leq k_{m}+1}\left(i^{2}+\right.$ 1) be such that $x \in \operatorname{int}\left(\bigcap_{2 \leq i \leq k_{m}+1} T_{i, s(i)}\right)$. For each $0 \leq j \leq m$, since $\operatorname{int}\left(\bigcap_{2 \leq i \leq k_{j}+1} T_{i}, s(i)\right) \neq \emptyset$, our construction of $X_{k_{j}}$ guarantees that $\exists x_{k_{j}} \in$ $X_{k_{j}} \cap \operatorname{int}\left(\bigcap_{2 \leq i \leq k_{j}+1} T_{i, s(i)}\right)$. Choose one such $x_{k_{j}}$. For $2 \leq i \leq N+2$, $X \cup\left\{x_{k}: k \in K^{\prime \prime}\right\}$ intersects exactly the same $T_{i j}$ 's as $X$; hence, $X \cup$ $\left\{x_{k}: k \in K^{\prime \prime}\right\}$ intersects less than $i$-many $T_{i j}$ 's. For $N+3 \leq i \leq k_{m}$, $\left\{x_{k}: k \in K^{\prime \prime}, k \geq i-1\right\}$ lies in $T_{i, s(i)}$, as does $x$. Hence, $X \cup\left\{x_{k}: k \in K^{\prime \prime}\right\}$ intersects at most $(i-2)$-many $T_{i j}$ 's. Since $\left|X \cup\left\{x_{k}: k \in K^{\prime \prime}\right\}\right| \leq k_{m}$, (a) holds for the sequence $\left\langle x_{k}: k \in K^{\prime \prime}\right\rangle$.

$Y \cap\left(\bigcup_{N<i<\omega} X_{i} \cup \bigcup_{N<i<\omega} F_{i}\right)=\emptyset$ and $K \cap L=\emptyset$. Hence, $\left(\bigcup_{k \in K^{\prime \prime}}\left(X_{k} \cup\right.\right.$ $\left.\left.F_{k}\right)\right) \cap\left(Y \cup \bigcup_{l \in L}\left(X_{l} \cup F_{l}\right)\right)=\emptyset . X \cap Y=\emptyset$, since $d>[\mathbf{0}] . X \cap\left(\bigcup_{2 \leq i<\omega} X_{i} \cup\right.$ $\left.F_{i}\right) \subseteq \bigcup_{i \in I}\left(X_{i} \cup F_{i}\right)$ and $I \cap L=\emptyset \Longrightarrow X \cap\left(\bigcup_{l \in L} X_{l} \cup F_{l}\right)=\emptyset$. Thus, (b) holds for the sequences $\left\langle f_{k}: k \in K^{\prime \prime}\right\rangle$ and $\left\langle x_{k}: k \in K^{\prime \prime}\right\rangle$. Thus, $c \wedge d \neq[\mathbf{0}]$.

Proposition 5.3 shows that $\mathbf{C}$ satisfies the conditions of Lemma 1.12. Hence, $\mathbf{C}$ is a regular subalgebra of $\mathbf{A}_{G}$. By Lemma 1.15, the Cohen algebra r.o. $\left(\mathbf{C}^{+}\right)$embeds as a complete subalgebra of $\mathbf{A}_{G}$.

\section{Remarks and acknowledgments.}

Remark 6.1. After the completion of the work in this paper, it was brought to our attention that A. Kamburelis has done some similar work on these three Boolean algebras in an unpublished paper $[\mathbf{K a}]$. Our work extends some and gives a new perspective on some of his results in that paper. Specifically, Kamburelis used forcing methods (in contrast to our constructive, purely Boolean-algebraic methods) to show that the weak $(\omega, \omega)$-d.l. fails in the Galvin-Hajnal, Agryros, and Gaifman algebras.

In this paper, Kamburelis mentions K. Skandalis' remark that his proof of the failure everywhere of the weak $(\omega, \omega)$-d.l. in the the Argyros algebra can be easily modified to produce a Cohen real. By the result of Shelah $[\mathbf{S}]$ mentioned in Remark 1.16, we now know that Kamburelis' proof of the failure everywhere of the weak $(\omega, \omega)$-d.l. actually implies the Argyros algebra 
adds a Cohen real. In addition, Kamburelis showed that the Gaifman algebra adds a Cohen real, although to do this, he assumed that the Gaifman algebra contains no atoms, which, as we showed, is not always the case.

Acknowledgment 6.2. We are enormously grateful to the referee for many detailed and helpful comments and suggestions. In particular, we would like to thank the referee for pointing out that our original proof that the Cohen algebra completely embeds into the Galvin-Hajnal algebra, in essence, showed that it actually adds $\operatorname{cf}\left(2^{\omega}\right)$-many Cohen reals, and for suggesting that we investigate the variants of Argyros' algebra.

Acknowledgment 6.3. Infinite gratitude goes to my thesis advisor K. Prikry for his idea of trying to completely embed the Cohen algebra into the Argyros, Galvin-Hajnal, and Gaifman algebras. Thanks also goes to him for pointing out the connections between von Neumann's problem and weak distributivity in c.c.c. Boolean algebras, and for many helpful discussions.

\section{References}

[A] S.A. Argyros, On compact spaces without strictly positive measure, Pacific J. Math., 105(2) (1983), 257-272, MR 0691601, Zbl 0523.54016.

[BJ] S.T. Bartoszyński and H. Judah, Set Theory on the Structure of the Real Line, A.K. Peters, Ltd., 1995, MR 1350295, Zbl 0834.04001.

[CN] W.W. Comfort and S. Negrepontis, Chain Conditions in Topology, Cambridge University Press, 1982, 162-166, MR 0665100, Zbl 0488.54002.

[F] D. Fremlin, Measure Algebras, Handbook of Boolean Algebra, 3, North-Holland, 1989, 877-980, MR 0991611, Zbl 0671.06001.

[Ga] H. Gaifman, Concerning measures on Boolean algebras, Pacific J. Math., 14 (1964), 61-73, MR 0161952, Zbl 0127.02306.

[GP] F. Galvin and K. Prikry, On Kelley's intersection numbers, Proc. Amer. Math. Soc., 129(2) (2001), 315-323, MR 1707516, Zbl 0961.06011.

[Gl] W. Glówczyński, Measures on Boolean algebras, Proc. Amer. Math. Soc., 111 (1991), 845-849, MR 1050019, Zbl 0718.03041.

[J] R. Jensen, Definable sets of minimal degree, Mathematical Logic and Foundations of Set Theory, Proc. Internat. Coll., Jerusalem 1968, North-Holland, 1970, 122-128, MR 0306002, Zbl 0245.02055.

[Ka] A. Kamburelis, On weak distributivity of some topological spaces. Preprint.

[Ke] J. Kelley, Measures on Boolean algebras, Pacific J. Math., 9 (1959), 1165-1177, MR 0108570, Zbl 0087.04801.

[Ko] S. Koppelberg, Handbook of Boolean Algebra, 1, North-Holland, 1989, MR 0991565, Zbl 0671.06001.

[Mah] D. Maharam, An algebraic characterization of measure algebras, Ann. Math., 48(1) (1947), 154-167, MR 0018718, Zbl 0029.20401.

[Mau] D. Mauldin, The Scottish Book, Birhäuser, 1981, MR 0666400, Zbl 0485.01013. 
[S] S. Shelah, How special are Cohen and random real forcings, i.e., Boolean algebras of the family of subsets of reals modulo meagre or null, Israel J. Math., 88 (1994), 159-174, MR 1303493, Zbl 0815.03031.

[V] B. Veličković, CCC posets of perfect trees, Comp. Math., 79 (1991), 279-294, MR 1121140, Zbl 0735.03023.

Received December 3, 2002 and revised July 22, 2003.

The Pennsylvania State University

Department of Mathematics

218 MCAllister Building

UNIVERsity PARK, PA 16802

E-mail address: dobrinen@math.psu.edu 